\title{
Ergonomics of novices and experts during simulated endotracheal intubation
}

\author{
Adam de Laveaga ${ }^{1}$, Michael C. Wadman ${ }^{2}$, Laura Wirth ${ }^{1}$ and M. Susan Hallbeck ${ }^{1}$ \\ ${ }^{1}$ Innovative Design and Ergonomic Analysis Laboratory, Industrial and Management Systems Engi- \\ neering, University of Nebraska, Lincoln, NE, USA; ${ }^{2}$ University of Nebraska Medical Center, Omaha, \\ NE, USA
}

\begin{abstract}
Endotracheal Intubation (ETI) is an airway procedure commonly used to secure the airway for a variety of medical conditions. Proficiency in ETI procedures requires significant clinical experience and insufficient data currently exists describing the physical ergonomics of successful direct laryngoscopy. The research objectives of this study were to examine how ETI time, error and practitioner biomechanics varied among clinical experience levels and hospital bed heights. The participant population included novice and expert personnel, differentiated by their exposure to ETI procedures. Participants used a standard laryngoscope and blade to perform ETI trials on an airway manikin trainer at predesigned hospital bed heights. Participants were evaluated based on ETI time and accuracy, as well as wrist postures and muscle utilization. Hospital bed height did not affect task completion time, error rates or muscle utilization. Expert participants exhibited less ulnar deviation and forearm supination during task trials, as well as a higher utilization of the bicep brachii and anterior deltoid muscles. Expert grasped instrumentation differently, requiring less wrist manipulation required to achieve ideal instrument positions. By encouraging ergonomic best-practices in hand and arm postures during ETI training, the opportunity exists to improve patient safety and reduce the learning curve associated with ETI procedures.
\end{abstract}

Keywords: Ergonomics, Laryngoscopy, Wrist Postures, EMG, Emergency Medicine, Biomechanics

\section{Introduction}

\subsection{ETI Overview}

Proficiency in endotracheal intubation (ETI) requires significant training and real world experience, as errors are frequent and potentially life threatening. Training, teamwork and difficult airway management are paramount concerns in the field of intubation training and practice [3]. Because of the inherently chaotic environment of emergent situations, personnel proficiency performing intubation procedures can range widely. ETI procedures are typically performed by nurse anesthetists and resident and attending physicians within anesthesiology and emergency medicine departments.

Specific postures and practitioner ergonomics are not universally defined within intubation training, and instruction typically follows the appren- ticeship model of "see one, do one, teach one" within a variety of medical fields. Identifying best practices in intubation biomechanics could shorten the learning curve, improve ETI success rates and reduce ventilation time in both emergent and controlled intubation situations while also providing objective metrics for intubation teaching and learning.

\subsection{ETI Procedures}

With the patient in the supine position, the clinician uses their right hand to maintain the patient's head, neck and atlanto-occipital joint positions while operating the laryngoscope with their left hand. Accurate placement of the endotracheal tube requires that the clinician be able to expose and visualize the vocal chords of the patient by placing the laryngoscope at the base of the epiglottis and applying force along the axis of the laryn- 
goscope. Once the vocal chords are exposed, the endotracheal tube is then inserted beyond the trachea under direct vision, until a mid-trachea depth is achieved. Mid-trachea depth varies based upon patient anatomy, however in adult patients this depth is typically $21-23 \mathrm{~cm}$ beyond the teeth. At this point, the endotraceal tube cuff is inflated, protecting the lungs from aspiration while allowing for ventilation to begin.

\subsection{ETI Complications \& Risks}

Intubation success rates, occurrence of errors and instances of complications vary significantly across patient conditions and intubation environments [9]. In a 2006 study, blunt trauma was the leading adverse event experienced by patients during ETI procedures [3]. Intubation failure rates of $.13 \%-.30 \%$ and difficulty rates of $1.10 \%-$ $3.80 \%$ exist even in the controlled environment of the operating room [4]. The consequences of intubation complications are most severe in respiratory failure situations, and the inability to intubate and ventilate in such situations often results in patient morbidity and mortality [1]. Other ETI related complications include aspiration, anoxic brain injury, and fluid or blood in the airway $[9,10]$.

\subsection{ETI Training}

Traditional intubation training involves a combination of didactic and experiential learning [11]. Clinicians acquire ETI skills within the scope of their respective medical functions, and it has been observed that the evaluation and definition of intubation proficiency varies among emergency medicine, paramedic, and anesthesiology training [8]. In a 2006 study of ETI success rates by emergency medical residents (EMRs), training periods averaged 86 intubations in clinical rotations and laboratory settings, and residents were capable of intubating $91.6 \%$ of their patients by their third attempt [3]. Errors during intubation training range from early struggles with mechanics and endotracheal tube placement to view-related issues near proficiency [8].

Improvements in skill acquisition and retention were observed based upon experiential versus guided learning with ETI [12]. Physical ergonom- ics and the effect of patient position have not been adequately addressed within the literature. Multiple studies have explored the movement, position and force transmitted through the laryngoscope [2]; however previous electromyography and biomechanical research have not been related to task performance. This pilot study has been designed to define muscle utilization and hand, arm and wrist positions of practitioners, and observe how these vary with changes in experience level and patient position.

\section{Methods}

\subsection{Participants}

Twenty novice and expert participants were recruited from the University of Nebraska Medical Center to participate in this study. Fifteen third and fourth-year medical students were recruited to participate as novice subjects based upon a lack of prior experience with ETI procedures and airway equipment. Five expert participants were selected from the department of Emergency Medicine. All expert participants had more than 5 years of experience in emergency airway management. All participants reported that they had not experienced any musculoskeletal injuries in their left hand, arm or shoulder within the past 12 months. The participants' mean (standard deviation) information is displayed in Table 1.

$$
\text { Table } 1
$$

Participant Information

\begin{tabular}{|c|c|c|c|c|}
\hline & & Male & Female & Overall \\
\hline \multirow{3}{*}{$\begin{array}{l}\stackrel{8}{z} \\
\text { Z Z }\end{array}$} & Age (Years) & $25.3(1.4)$ & $23.8(1.9)$ & $24.7(1.8)$ \\
\hline & Height $(\mathrm{cm})$ & $178.6(5.1)$ & $163.0(3.5)$ & $171.8(9.1)$ \\
\hline & Weight $(\mathrm{kg})$ & $177.3(28.5)$ & $125.6(12.4)$ & $154.7(34.6)$ \\
\hline \multirow{3}{*}{ 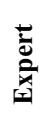 } & Age (Years) & $49.5(6.0)$ & $36.0(\mathrm{n} / \mathrm{a})$ & $46.8(8.6)$ \\
\hline & Height $(\mathrm{cm})$ & $179.8(7.4)$ & $186(\mathrm{n} / \mathrm{a})$ & $181.0(7.9)$ \\
\hline & Weight $(\mathrm{kg})$ & $174.3(15.8)$ & $300(\mathrm{n} / \mathrm{a})$ & $199.4(58.4)$ \\
\hline
\end{tabular}

\subsection{Apparatus \& Approach}

All ETI trials were completed in the Clinical Simulation Laboratory at the University Nebraska Medical Center. Simulated ETI procedures were completed using a Difficult Airway Trainer (Laerdal, Wappingers Falls, NY) situated in a supine position atop an adjustable-height hospital bed 
(Advanta, Hill-Rom Services, Inc., Batesville, IN). The trials took place at the maximum and minimum heights of the adjustable bed $(96 \mathrm{~cm}$ and 62 $\mathrm{cm})$. An assisting researcher held the forehead and abdomen of the airway manikin to resist lifting forces during each trial. A standard C-Cell laryngoscope (Rüsch Model No. 8321000, TeleFlex Medical Inc.), McIntosh No. 4 blade and size 7.0 endotracheal tube and stylet were used to complete the ETI procedures.

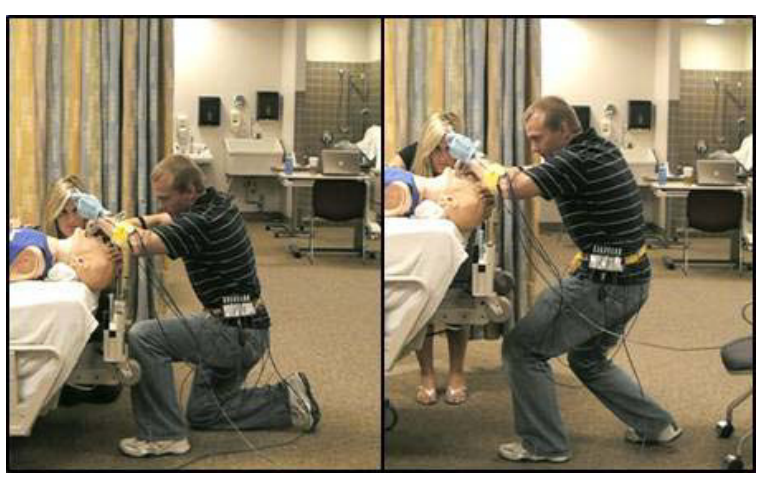

Fig. I - ETI Trial at $62 \mathrm{~cm}$ (left) and $96 \mathrm{~cm}$ (right)

Wrist positions and muscle activity of the left arm were measured during ETI trials to determine the effect of participant experience and patient position in relation to muscle utilization and wrist postures. Researchers chose to observe wrist flexion and extension, radial and ulnar deviation, and forearm pronation and supination to define the range of motion during ETI procedures.

Wrist posture data was gathered using a dualaxis goniometer (model No. SG150, Biometrics Ltd.) and torsiometer (model No. Q110, Biometrics Ltd., Gwent, UK). Researchers selected the flexor carpi radialis (FCR), extensor carpi radialis (ECR), bicep brachii and anterior deltoid muscles to evaluate grasping and lifting muscles associated with the task. Muscle utilization was recorded using bipolar surface electromyography (EMG) sensors (SX230, Biometrics Ltd., Gwent, UK), aligned along the center of each muscle and sampled at a rate of $1000 \mathrm{~Hz}[7,16]$. A digital event marker was utilized to record trial events. All equipment was connected to an eight-channel Biometrics DataLINK ${ }^{\mathrm{TM}}$ DLK900 system and recorded using DataLINK PC Software (V. 7.0, Biometrics Ltd., Gwent, UK).

\subsection{Experimental Procedure}

All subjects were informed of the purpose of the study, procedures and associated risks prior to participation and voluntarily signed informed consent and waiver agreements. Anthropometric measurements of stature, acromial height, elbow height, xiphoid process height, upper-arm length, forearm length, hand length, hand breadth, hand spread and grip span were recorded for all participants using a GPM $^{\mathrm{TM}}$ anthropometer, sliding digital calipers and a conical measuring device, respectively. After gathering anthropometric data, each participant responded to a brief questionnaire regarding areas of medical interest and ETI procedure perceptions and experience.

Participants were then seated and prepped for EMG sensor application using a sterile alcoholbased swab. Surface EMG sensors were individually placed over the ECR, FCR, bicep brachii and anterior deltoid muscles and fixed with a temporary two-way adhesive and athletic tape. Muscle location was determined using anthropometric landmarks [7] as well as active data from the EMG sensor and DataLINK ${ }^{\mathrm{TM}}$ software. Resting and maximum static contraction values were recorded for each observed muscle so that muscle utilization during the task could be normalized against a maximum impulse level specific to each participant.

To position the goniometer, each participant sat with their left hand and arm resting on a table and their palm resting naturally against the surface. The dual-axis goniometer was positioned along the second metacarpal, on top of the wrist and along the back side of the left hand. The torsiometer was positioned alongside the goniometer, extending along the forearm. To ensure a neutral starting position, the torsiometer was placed while the participant extended their left arm along the surface of the table, rotating their forearm into a neutral position alongside the vertical face of a box on the tabletop. Once positioned, the goniometer and torsiometer were calibrated for these neutral positions. Fig II displays the placement of goniometer and torsiometer sensors on the left hand and arm during a trial. 


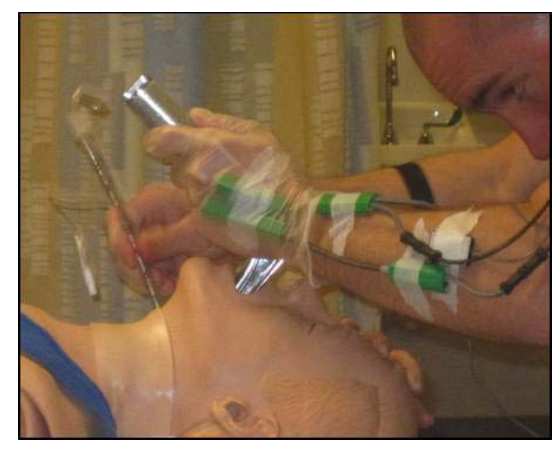

Fig. II - Location of goniometer and torsiometer sensors on left hand and arm

\subsection{Trial Procedure}

Our novice subgroup was unfamiliar with emergency airway management, ETI procedures and laryngoscopy equipment. Prior to performing task trials, each novice participant received a brief overview of airway anatomy, the airway trainer, standard terms in ETI procedures, step-by-step instructions for completing ETI tasks, and an introduction to laryngoscopy equipment. Participants also observed one example ETI procedure performed by the researchers. Participants were then made aware of what data would be recorded for each trial as well as specific errors to avoid during the task. Prior to trial commencement, each novice participant was allowed a single practice trial to familiarize themselves with the trainer and task, and to clarify any areas of uncertainty.

Task time was assessed as the time between the introduction of the laryngoscope and the secure placement of the endotracheal tube. Participants were responsible for determining correct endotracheal tube depth and notifying researchers when they felt confident that the trial was complete. After each trial, researchers would evaluate trial duration, endotracheal tube depth and placement, the number of intubation attempts, and any incidents of dental trauma. Participants were also asked to assess the achieved laryngeal view using the Cormack and Lehane (C \& L) scale [5].

Novice participants completed six total trials, three at each hospital bed height $(62 \mathrm{~cm}$ and $96 \mathrm{~cm})$. The starting order for bed height was randomized between participants. Each participant received a 60 second break between trials, and a five-minute break between trials three and four while bed height was adjusted. After trial 6, all participants completed additional task-related qualitative questions regarding ETI procedures before being released from the study.

Expert participants acknowledged proficiency with ETI procedures and were only acquainted with the airway trainer and study objectives. Due to the limited availability of expert participants, as well as a mild learning effect noticed during novice data collection, the trial procedure for expert participants was modified to improve the reliability of trial data. Expert participants completed five ETI trials at each hospital bed height starting with 96 $\mathrm{cm}$ and ending at $62 \mathrm{~cm}$, for a total of 10 trials. Due to the limited sample size, the starting height was not randomized for the expert subgroup. Expert participants received 60 -second rest breaks between trials and a five-minute break between the 96 $\mathrm{cm}$ and $62 \mathrm{~cm}$ trial sets. Expert participants completed a similar pre and post-procedure questionnaire adapted for their skills and experience with ETI procedures. All trial performance metrics were consistent between expert and novice participants.

\subsection{Data Analysis}

Trial data was trimmed using DataLINK ${ }^{\mathrm{TM}}(\mathrm{V}$. 7.0) PC Software to contain only data from the trial time period dictated by inputs from the digital event marker. EMG data channels were adjusted using Add-for-Zero and root mean square (RMS) filters to provide the absolute value of muscle activity within the trials. The mean muscle utilization figure for each muscle during task trials was divided by the average maximum contraction value (specific to each participant), resulting in a proportion of muscle utilization per muscle and trial. Variable data from goniometer and torsiometer readings required no additional processing and was adjusted for task duration only.

Categorical variables were created to describe wrist postures and muscle utilization using a fiveclass frequency distribution with equal group densities. These categorized variables allowed researchers to perform additional ANOVA tests. The frequency distributions represent the entire range of data obtained for each variable, and specific cut points for each data set were determined using SPSS (V. 19.0) statistical software. 


\subsection{Experimental Design}

Several analyses were constructed to determine the influence of wrist postures, muscle utilization and hospital bed height within and between the novice and expert subgroups. Initial ANOVA tests were run on the dependent variable of task time by blocking on participant subgroups to determine the significant main effects among all variables. Independent variables for these analyses included the trial number, bed height, gender, starting order and the categorical variables for all EMG and wrist posture data. Post hoc (Tukey) tests for nonhomogeneity were then performed for statistically significant factors to determine the mean differences between treatments of independent variables. Additionally, paired and independent-sample t-tests were also used to examine the significance of bed height and participant type on a variety of dependent variables.

To examine muscle utilization and wrist postures between subgroups and hospital bed heights (an interaction), this interaction variable 'permutation' was created to define trials belonging to expert and novice participants at the two different bed heights. Simple one-way ANOVA and Tukey tests were then performed using the continuous EMG and wrist posture data as the dependent variable and the trial permutation as the independent variable. All analyses were performed using SPSS (V. 19.0) statistical software.

\section{Evaluation of Experiment Results}

\subsection{Task-Related Statistics}

Novice participants required greater time to complete ETI procedures than expert participants while also making significantly more errors ( $\mathrm{p} \leq$ 0.050). Neither group exhibited significant changes in error rates at lower bed heights, however novice trials included more errors at a near-significant level $(p=0.058)$. Expert participants required less time to complete trials at the lower bed height $(\mathrm{p}=$ 0.007 ), although the actual time differences between heights were minimal. Additionally, differences in laryngeal view and endotracheal tube placement accuracy were not significant between subgroups or bed heights. Task-related statistics are displayed in Table 2 .
Table 2

\begin{tabular}{|lllll|}
\hline \multicolumn{5}{|c}{ ETI Trial Statistics } \\
& Time (sec) & Depth $(\mathrm{cm})$ & Error & $\begin{array}{l}\text { C \& L } \\
\text { View }\end{array}$ \\
\hline Exp. 96 cm & $10.2(2.7)$ & $22.1(0.8)$ & $8 \%$ & 2.04 \\
Nov. 96 cm & $21.0(8.9)$ & $21.7(1.2)$ & $45 \%$ & 2.02 \\
Exp. 62 cm & $8.7(2.5)$ & $21.44(0.9)$ & $16 \%$ & 2.08 \\
Nov. 62 cm & $18.4(6.9)$ & $21.9(0.7)$ & $31 \%$ & 2.02 \\
\hline
\end{tabular}

Among novice participants, the main effects and two-way interactions of gender, trial number and bed height were significantly related to task completion time $(\mathrm{p} \leq 0.050)$. The independent variables of starting order and all categorical values for EMG and wrist posture data were not significantly related to task completion time $(p>0.050)$.

Among expert participants, only the main effect of trial number was significantly related to task completion time $(p=0.028)$. The main and twoway interaction effects of bed height, gender and all categorical values for wrist postures and EMG data were not significantly related to task completion time $(p>0.050)$.

The lack of significant main effects among experts with respect to time can be partially explained by the minimal variation in task completion times among experts (mean $=9.44$ sec., standard deviation = 2.71). Fig III illustrates the difference in task completion time and the effect of trial sequence among novice and expert participants sharing identical trial sequencing (the first 3 trials were used from each level for expert participants).

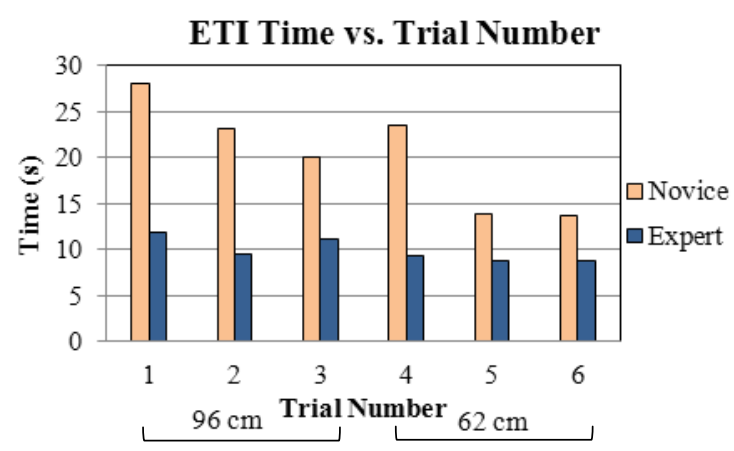

Fig. III - Trial completion time by trial number, height and subgroup

\subsection{Wrist Postures}

Wrist posture data (degrees) was analyzed using the independent variables of subject type and 
bed height. The main effects of subject type were significant for wrist extension $(p=0.009)$, ulnar deviation $(\mathrm{p}<0.001)$, and forearm supination $(\mathrm{p}=$ 0.002). Additionally, bed height did not reveal any significant effects among wrist posture data. To further examine differences within treatments of each variable, an interaction variable was created to define the trial permutation of participant subgroup and hospital bed height. Statistically significant effects were observed between the interaction variable and wrist extension ( $p=0.007)$, and ulnar deviation $(\mathrm{p}<0.001)$, and forearm supination data $(\mathrm{p}=$ 0.008 ). Mean differences between treatments were analyzed using a Tukey test and significant differences are designated by groupings (A, B) in Fig. IV.

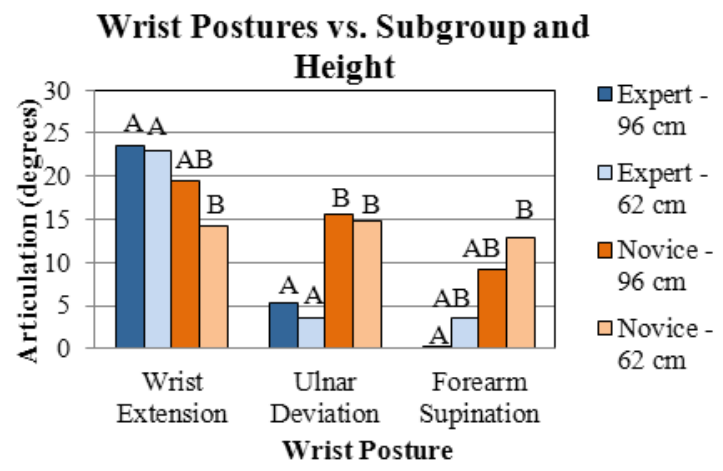

Fig. IV - The effect of subgroup and bed height on wrist postures

\subsection{Muscle Utilization}

Utilization of the ECR, FCR, bicep brachii and anterior deltoid (\% of maximum) were examined using the independent variables of subject type and hospital bed height. In all cases, muscle utilization was not different for changes in the hospital bed height variable $(p>0.050)$. Highly significant effects were found for the variation in bicep brachii $(\mathrm{p}<0.001)$ and anterior deltoid $(\mathrm{p}=0.001)$ utilization between novice and expert subject groups. Significant differences did not exist between ECR and FCR utilization between participant groups. The interaction variable was used to investigate the effect of participant subgroup and hospital bed height by muscle utilized. The effects of the interaction variable against the dependent variables of ECR and FCR utilization were not statistically significant $(\mathrm{p}>0.050)$, however significant effects were observed for bicep brachii and anterior deltoid utilization $(\mathrm{p}=0.006, \mathrm{p}=0.010)$. Muscle utiliza- tion across participant subject types and hospital bed heights are shown in Fig. V.

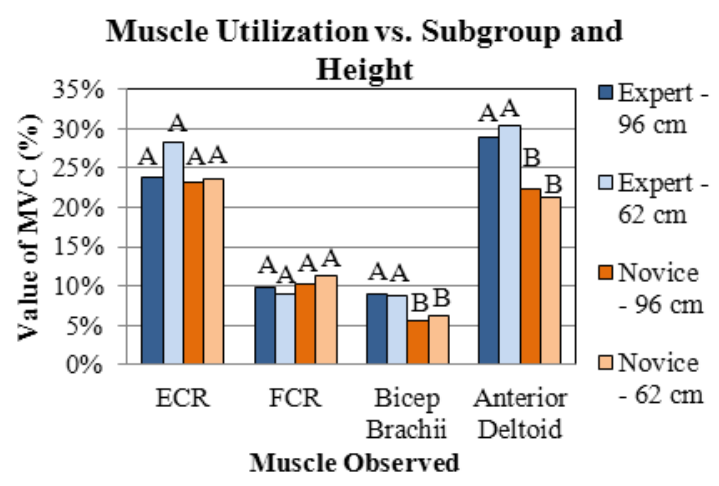

Fig. V - The effect of subgroup and hospital bed height on muscle utilization

\section{Discussion}

The purpose of this study was to examine the relationship between experience level, patient position (hospital bed height), muscle utilization and wrist postures with respect to the completion of ETI procedures. Because of the variety of circumstances requiring endotracheal tube placement, researchers were hoping to provide substantial evidence that proper patient positioning would reduce the amount of time required to perform ETI procedures, while also reducing task-related errors. Additionally, researchers expected to see significant differences for wrist postures and muscle utilization between novice and expert subject groups.

A non-significant reduction in errors was observed at the higher bed heights among expert participants; however this did not hold true for novice participants. Minor variations in muscle utilization existed between subject groups; expert participants used a greater proportion of their bicep brachii and anterior deltoid muscles. Utilization of the forearm muscles did not differ between subject groups, and bed height had a minimal effect on levels of muscle utilization within subject groups. Based upon individual maximums, the data in this study indicates that clinicians use approximately $5 \%-30 \%$ of their maximum output of grasping and lifting muscles during simulated ETI procedures. Additionally, initial data indicates that successful clinicians rely upon a high level of large-muscle utilization in 
their left arm. This finding supports previous research regarding the importance of lifting force during successful endotracheal tube placement [2].

Expert participants exhibited significantly less ulnar deviation and forearm supination, as well as greater wrist extension when compared to novice participants, while achieving similar orientations of the laryngoscope blade and handle. The significant findings form this this data supports the observation that experienced practitioners grasp the laryngoscope in-line with their forearm and across their palm, rather than gripping the handle normal (perpendicular in orientation) to the forearm. As a result, fewer awkward wrist and forearm postures were observed for the experienced practitioners as they performed ETI procedures, resulting in greater recruitment of appropriate lifting muscles and a reduction in task completion time. Applications of these findings could contribute to the improvement of ETI procedure training within medical residencies as well as a preliminary study for an ergonomic redesign of the laryngoscope handle to improve the intuitive hand, arm and tool relationships.

\section{Acknowledgements}

The authors wish to acknowledge Patricia Carstens and other members of the University of Nebraska Medical Center- Sorrell Clinical Simulation Lab for their assistance in this study.

\section{References}

[1] Berkow, L. C., Greenberg, R. S., Kan, K. H., Colantuoni, E., Mark, L. J., Flint, P. W., . . Heitmiller, E. S. (2009). Need for emergency surgical airway reduced by a comprehensive difficult airway program. Anesthesia and Analgesia, 109(6), 1860-1869. doi:10.1213/ane.0b013e3181b2531a

[2] Bucx, M. J., van Geel, R. T., Scheck, P. A., Stijnen, T., \& Erdmann, W. (1992). Forces applied during laryngoscopy and their relationship with patient characteristics. influence of height, weight, age, sex and presence of maxillary incisors. Anaesthesia, 47(7), 601-603.

[3] Casey, Z. C., Smally, A. J., Grant, R. J., \& McQuay, J. (2007). Trauma intubations: Can a protocol-driven approach be successful? The Journal of Trauma, 63(4), 955960. doi:10.1097/TA.0b013e3181271b4c

[4] Crosby, E. T., Cooper, R. M., Douglas, M. J., Doyle, D. J., Hung, O. R., Labrecque, P., . . Roy, L. (1998). The unanticipated difficult airway with recommendations for management. Canadian Journal of Anaesthesia = Journal Canadien d'Anesthesie, 45(8), 757-776.

[5] Krage, R., van Rijn, C., van Groeningen, D., Loer, S. A., Schwarte, L. A., \& Schober, P. (2010). Cormack-lehane classification revisited. British Journal of Anaesthesia, 105(2), 220-227. doi:10.1093/bja/aeq136

[6] Mulcaster, J. T., Mills, J., Hung, O. R., MacQuarrie, K., Law, J. A., Pytka, S., . . Field, C. (2003). Laryngoscopic intubation: Learning and performance. Anesthesiology, 98(1), 23-27.

[7] Perotto, A. O., M.D. (2005). Anatomical guide for the electromyographer. Springfield, Illinois: Charles C. Thomas Publisher, Ltd.

[8] Reed, M. J. (2007). Intubation training in emergency medicine: A review of one trainee's first 100 procedures. Emergency Medicine Journal : EMJ, 24(9), 654-656. doi:10.1136/emj.2007.048678

[9] Smale, J. R., Kutty, K., Ohlert, J., \& Cotter, T. (1995). Endotracheal intubation by paramedics during in-hospital CPR. Chest, 107(6), 1655-1661.

[10]Smith, C. E., \& Dejoy, S. J. (2001). New equipment and techniques for airway management in trauma. Current Opinion in Anaesthesiology, 14(2), 197-209.

[11] Stewart, R. D., Paris, P. M., Pelton, G. H., \& Garretson, D. (1984). Effect of varied training techniques on field endotracheal intubation success rates. Annals of Emergency Medicine, 13(11), 1032-1036.

[12]Ti, L. K., Chen, F. G., Tan, G. M., Tan, W. T., Tan, J. M., Shen, L., \& Goy, R. W. (2009). Experiential learning improves the learning and retention of endotracheal intubation. Medical Education, 43(7), 654-660. doi:10.1111/j.13652923.2009.03399.x

[13] University of Virginia School of Medicine. (2004). Airway management: Understanding equipment. Retrieved 3/5/2010, 2010, from

http://www.healthsystem.virginia.edu/Internet/Anesthesiolo gy-Elective/airway/equipment.cfm

[14] Walls, R., Barton, E., \& McAfee, A. (1999). 2,392 emergency department intubations: First report of the ongoing national emergency airway registry study (near 97). Annals of Emergency Medicine, 26, 364-403.

[15] Walls, R. M., Brown, C. A.,3rd, Bair, A. E., Pallin, D. J., \& of the NEAR II Investigators. (2010). Emergency airway management: A multi-center report of 8937 emergency department intubations. The Journal of Emergency Medicine, doi:10.1016/j.jemermed.201

[16]Zipp, P., (1982). Recommendations for the standardization of lead positions in surface electromyography. European Journal of Applied Physiology and Occupational Physiology, $41-5$. 